\title{
LIMITS OF COMPLETE HOLOMORPHIC VECTOR FIELDS
}

\author{
FRANC ForstneRIC
}

\begin{abstract}
A в stract. Let $V$ be a holomorphic vector field on a Stein manifold $M$. If $V$ can be approximated by complete holomorphic vector fields, uniformly on compacts in $M$, we prove that the fundamental domain of $V$ is single sheeted, pseudoconvex, and it has simply connected fibers. Moreover, every complex orbit of $V$ has connectivity at most one (Theorem 1.1). We then find several explicit classes of holomorphic vector fields on $\mathbf{C}^{2}$ which are not limits of complete fields (Corollaries 1.4-1.6).
\end{abstract}

\section{Introduction and results}

Recently Buzzard and Fornaess answered the question, raised in [8], as to whether there exist holomorphic vector fields on $\mathbf{C}^{n}$ for $n \geq 2$ which can not be approximated by complete holomorphic vector fields. They showed for instance that the quadratic vector field on $\mathbf{C}^{2}, V(z, w)=z(z-1) \frac{\partial}{\partial z}-w \frac{\partial}{\partial w}$, is not a limit of complete holomorphic fields; the same is true for the field $z^{2} \frac{\partial}{\partial z}$ on $\mathbf{C}^{2}$ (private communication, January 1995). Their method proves that the set of complete holomorphic vector fields is nowhere dense in the set of all entire vector fields on $\mathbf{C}^{n}$, in the topology of uniform convergence on compact sets. It was clear from the results of [8] that most Hamiltonian holomorphic vector fields can not be approximated by complete Hamiltonian fields. This phenomenon is in strong contrast with the situation for smooth vector fields which can always be made complete by a modification supported outside a given compact set in $M$.

In this article we obtain qualitative information on the fundamental domain in complex time of those holomorphic vector fields $V$ on a Stein manifold $M$ which are limits of complete fields (Theorem 1.1). We apply this to show that several specific types of vector fields on $\mathbf{C}^{2}$ are not limits of complete fields (uniformly on compacts).

Received March 13, 1995.

Supported in part by the NSF grant DMS-9322766 and by a grant from the Ministry of Science of the Republic of Slovenia. 
In order to define the fundamental domain we consider the ordinary differential equation associated to the field $V$ :

$$
\dot{Z}=V(Z), \quad Z(0)=z \in M \text {. }
$$

This equation has a unique local solution $t \mapsto \phi^{z}(t)=\phi(t, z)$, defined and jointly holomorphic for $(t, z)$ in an open neighborhood of $\{0\} \times M \subset \mathbf{C} \times M$, satisfying $\phi(t, \phi(s, z))=\phi(t+s, z)$ where both sides are defined. We call $\phi$ (and also $\phi^{z}$ ) the (local) flow of $V$.

We fix a point $z \in M$ and extend the local solution $\phi^{z}$ of (1) by analytic continuation along paths in $\mathbf{C}$, originating at $t=0$, to a maximal connected Riemann surface $\pi_{z}: \Omega_{z} \rightarrow \mathbf{C}$ which is spread as a Riemann domain over $\mathbf{C}$. $\pi_{z}$ is the natural base point projection, i.e., points in $\Omega_{z}$ lying over $t \in \mathbf{C}$ are obtained by analytic continuation of $\phi^{z}$ along paths in $\mathbf{C}$ which begin at 0 and end at $t$, and $\phi^{z}$ separates points on each fiber of $\pi_{z}$.

\section{Definition.}

(a) The Riemann surface $\pi_{z}: \Omega_{z} \rightarrow \mathbf{C}$ is called the maximal domain of the flow of $V$ through the point $z \in M$, and its image $C_{z}=$ $\phi^{z}\left(\Omega_{z}\right) \subset M$ is called the complex orbit of $V$ through $z$.

(b) The fundamental domain of $V$ (and of its flow) is the disjoint union of the $\Omega_{z}$ 's:

$$
\Omega=\Omega^{V}=\bigcup_{z \in M} \Omega_{z} \times\{z\} .
$$

(c) $V$ is complete (in real time) if $\Omega_{z}$ contains the real axis $\mathbf{R}$ (i.e., if $\phi^{z}$ extends to all $t \in \mathbf{R}$ ) for every $z \in M$.

(d) $V$ is complete in complex time if $\Omega_{z}=\mathbf{C}$ for every $z \in M$.

In general the Riemann domain $\pi_{z}: \Omega_{z} \rightarrow \mathbf{C}$ may be multiply sheeted, and it need not be simply connected (see the examples below). $\Omega$ has a natural structure of a complex manifold such that the base point projection

$$
\pi: \Omega \rightarrow \mathbf{C} \times M, \quad \pi(t, z)=\left(\pi_{z}(t), z\right)
$$

is holomorphic and spreads $\Omega$ as a Riemann domain over $\mathbf{C} \times M$. When $\pi$ is single sheeted, we shall identify $\Omega$ with the domain $\pi(\Omega) \subset \mathbf{C} \times M$.

When $M$ is a Stein manifold, we can also define $\Omega$ as follows. Let

$$
\pi: \tilde{\Omega} \rightarrow \mathbf{C} \times M
$$

be the maximal Riemann domain to which the local flow $\phi$ of $V$ can be extended by joint analytic continuation in the variables $(t, z)$. Such a maximal domain always exists and it is a Stein manifold [11]. For each $z \in M$ 
let $\Omega_{z}$ be the connected component of the fiber $\tilde{\Omega}_{z}$ containing the origin $t=0$, and define $\Omega$ to be the disjoint union of the $\Omega_{z}$ 's as above.

Holomorphic vector fields which are conjugate by a holomorphic automorphism clearly have biholomorphically equivalent fundamental domains.

Our main result is

1.1 Theorem. If $M$ is a Stein manifold and $V$ is a holomorphic vector field on $M$ which is a limit of complete holomorphic vector fields, uniformly on compacts in $M$, then for each $z \in M$ the maximal domain $\pi_{z}: \Omega_{z} \rightarrow \mathbf{C}$ of the flow is single sheeted and simply connected, and the complex orbit $C_{z}=\phi^{z}\left(\Omega_{z}\right) \subset M$ is biholomorphic to one of the surfaces $\mathbf{C}, \mathbf{C}^{*}=\mathbf{C} \backslash\{0\}$, $U$ (=the disc), $U^{*}=U \backslash\{0\}$, or an annulus. Moreover, the fundamental domain $\Omega \subset \mathbf{C} \times M(2)$ is pseudoconvex.

At this time we do not know whether there are other obstructions to approximation of holomorphic vector fields by complete fields. Explicitly, if Theorem 1.1 is satisfied by the fundamental domain of a holomorphic vector field $V$, is $V$ a limit of complete holomorphic vector fields?

Notice that the obstructions to approximation by complete fields, given by Theorem 1.1, can not be removed by extending $V$ to some larger complex manifold containing $M$.

For later reference we recall from [8] (Proposition 2.1) that the fundamental domain of an $\mathbf{R}$-complete holomorphic vector field on any complex manifold $M$ is of the form

$$
\Omega=\{(t, z): t \in \mathbf{C}, z \in M,-b(z)<\Im t<a(z)\}
$$

for some lower semicontinuous functions $a, b: M \rightarrow(0, \infty]$ which are constant if and only if they are equal to $+\infty$. Every complex orbit of $V$ is biholomorphic to one of the surfaces listed in Theorem 1.1 or to a complex torus. Clearly $V$ is $\mathbf{C}$-complete if and only if $a=b=+\infty$. If $M$ is Stein, then the functions $-a,-b: M \rightarrow[-\infty, 0)$ are plurisubharmonic or identically $-\infty$. If $M$ supports no nonconstant bounded plurisubharmonic functions, it follows that $a=b=\infty$, and hence every R-complete holomorphic vector field on $M$ is also C-complete ([8], Corollary 2.2). This holds for instance when $M=\mathbf{C}^{n}$.

Theorem 1.1 implies that either $\Omega_{z}=\mathbf{C}$ or else $\Omega_{z}$ is conformally equivalent to the disc. If $\Omega_{z}=\mathbf{C}$ for all points $z$ in a non-pluripolar subset $E \subset M$, it follows from a classical theorem of Hartogs that $\Omega_{z}=\mathbf{C}$ for all $z \in M[3]$, and hence $V$ is $\mathbf{C}$-complete.

The proof of Theorem 1.1 is given in section 2 . We now give examples and corollaries. 
Example 1. Every connected Riemann domain $\pi: R \rightarrow \mathbf{C}$ over $\mathbf{C}$ satisfying $0 \in \pi(R)$ can be realized as a maximal domain of the flow of a holomorphic vector field $V$ on $\mathbf{C}^{3}$. (Recall from [10] that every open Riemann surface $R$ admits an immersion $\pi: R \rightarrow \mathbf{C}$ into $\mathbf{C}$.)

To see this, we pull back the constant vector field $\frac{\partial}{\partial t}$ on $\mathbf{C}$ by the immersion $\pi$ to get a holomorphic vector field on $R$ which we shall still denote by $\frac{\partial}{\partial t}$. Suppose that $M$ is a Stein manifold and $f: R \rightarrow M$ is a proper holomorphic embedding. Such an embedding always exists into $\mathbf{C}^{n}$ for $n \geq 3$ [11]. If $R$ is a finitely connected domain in $\mathbf{C}$ then such an embedding also exists into $\mathbf{C}^{2}$ (Globevnik and Stensønes [9]). The push forward $V(f(t))=D f(t) \frac{\partial}{\partial t}$ is a holomorphic vector field on the embedded complex curve $f(R)=C \subset M$. By Cartan's Theorem A [11] we can extend $V$ from $C$ to a holomorphic vector field on all of $M$. Clearly $C$ is a complex orbit of the extended field $V$, with the corresponding maximal domain $\pi: R \rightarrow \mathbf{C}$.

If the projection $\pi: R \rightarrow \mathbf{C}$ is not single sheeted, or if the Riemann surface $R$ is not simply connected, then the field $V$ is not a limit of complete fields on $M$ according to Theorem 1.1.

With a more precise argument along these lines we obtain

1.2 Proposition. Let $M$ be a Stein manifold of dimension $\geq 2, W$ a holomorphic vector field on $M, K$ a compact subset of $M$, and $z \in M \backslash \hat{K}$. Then there exist holomorphic vector fields $V$ on $M$ which are arbitrary close to $W$ on $K$ and such that the maximal domain $\pi_{z}: \Omega_{z} \rightarrow \mathbf{C}$ of the flow of $V$ through the point $z$ is not single sheeted.

Here, $\hat{K}$ denotes the hull of $K$ with respect to the algebra of all holomorphic functions on $M$. One can do the same for a finite number of points $z_{1}, \ldots, z_{k} \in M \backslash \hat{K}$.

Proof. Since $z \in M \backslash \hat{K}$, there exist holomorphic functions $f$ on $M$ such that $f(z)=0$ and $f$ has no zeros on $\hat{K}$. For a generic choice of an $(n-1)$ tuple of such functions $f_{1}, \ldots, f_{n-1}$ (where $n=\operatorname{dim} M$ ), the map $F=$ $\left(f_{1}, f_{2}, \ldots, f_{n-1}\right): M \rightarrow \mathbf{C}^{n-1}$ has no critical points on $F^{-1}(0)$. Let $C$ be the connected component of $F^{-1}(0)$ containing $z$. Then $C$ is a closed, smooth complex curve in $M$.

There exists a holomorphic immersion $\pi: C \rightarrow \mathbf{C}$ into $\mathbf{C}$ such that $\pi(z)=$ 0 [10]. Replacing $\pi$ by the immersion $e^{\lambda \pi}-1$ for a sufficiently large $\lambda>0$ we may assume that $\pi$ is not single sheeted. Let $X$ be the unique holomorphic vector field on $M$, defined along $C$ and tangent to $C$, such that $D \pi \cdot X=$ $\frac{\partial}{\partial t}$ is the coordinate vector field on $\pi(C) \subset \mathbf{C}$. We can extend $X$ to a holomorphic vector field on all of $M$ by the standard techniques, using Cartan's theorems A and B [11]. By construction the immersion $\pi: C \rightarrow \mathbf{C}$ is precisely the maximal domain of the flow of $X$ through the point $z$. 
It remains to modify $X$ outside $C$ to obtain a holomorphic vector field $V$ which approximates a given field $W$ in a neighborhood of $\hat{K}$. We seek $V$ in the form

$$
V=X+\sum_{j=1}^{n-1} f_{j} X_{j}
$$

for suitably chosen holomorphic vector fields $X_{j}(1 \leq j \leq n-1)$. Since at each point of $\hat{K}$ at least one of the functions $f_{j}$ is nonzero, a standard application of Cartan's theorem B gives holomorphic vector fields $\tilde{X}_{j}(1 \leq$ $j \leq n-1)$ in a neighborhood of $\hat{K}$ such that $X+\sum f_{j} \tilde{X}_{j}=W$ there. Approximating the $\tilde{X}_{j}$ 's close enough near $\hat{K}$ by globally defined fields $X_{j}$ for $1 \leq j \leq n-1$ finishes the job.

The following proposition is likely not original, but we nevertheless include a simple proof in section 2 .

1.3 Proposition. If $V$ is an entire vector field on $\mathbf{C}$ such that, for some $z \in \mathbf{C}, V(z) \neq 0$ and the maximal domain $\pi_{z}: \Omega_{z} \mapsto \mathbf{C}$ of the flow of $V$ through $z$ is simply connected and single sheeted, then the vector field $V$ is affine linear: $V(z)=(\lambda z+c) \frac{\partial}{\partial z}$.

1.4 Corollary. Let $V$ be a decoupled entire vector field on $\mathbf{C}^{2}$ :

$$
V(z, w)=P(z) \frac{\partial}{\partial z}+Q(w) \frac{\partial}{\partial w} .
$$

If $V$ is not affine linear and if at least one of the components $P, Q$ has a zero in $\mathbf{C}$, then $V$ is not a limit of complete holomorphic vector fields on $\mathbf{C}^{2}$.

Proof. We may assume that $Q$ has a zero, $w_{0}$. Assume first that $P$ is not affine linear. Choose a point $z_{0}$ such that $P\left(z_{0}\right) \neq 0$. The maximal domain $\Omega_{p} \mapsto \mathbf{C}$ of $V$ through the point $p=\left(z_{0}, w_{0}\right)$ then coincides with the maximal domain of the one variable field $P(z) \frac{\partial}{\partial z}$ through $z_{0}$. Since $P$ is not affine linear, $\Omega_{p}$ is not single sheeted and simply connected according to Proposition 1.3. Hence Theorem 1.1 implies that $V$ is not a limit of complete fields.

If $P$ is affine linear, then $Q$ is not. If $P$ has a zero, $z_{0}$, then the previous proof applies with the reversed roles of $P$ and $Q$. In the remaining case when $P(z)=c$ is a constant, its flow is $(t, z) \rightarrow z+c t$. Hence the maximal domain of $V$ through any point $(z, w) \in \mathbf{C}^{2}$ is equal to the maximal domain of the one variable field $Q(w) \frac{\partial}{\partial w}$ through $w$. Since $Q$ is not affine linear, Proposition 1.3 and Theorem 1.1 apply as before. Hence, Corollary 1.4 is proven.

The same argument gives 
1.5 Corollary. Let $V$ be a holomorphic vector field on $\mathbf{C}^{2}$ of the form

$$
V(z, w)=P(z) \frac{\partial}{\partial z}+\left(w-w_{0}\right) Q(z, w) \frac{\partial}{\partial w},
$$

where $P$ (resp. $Q$ ) are entire functions on $\mathbf{C}$ (resp. $\mathbf{C}^{2}$ ) and $P$ is not affine linear. Then $V$ is not a limit of complete holomorphic vector fields on $\mathbf{C}^{2}$.

In another direction we consider Hamiltonian holomorphic vector fields on $\mathbf{C}^{2}$,

$$
X_{H}=\frac{\partial H}{\partial w} \frac{\partial}{\partial z}-\frac{\partial H}{\partial z} \frac{\partial}{\partial w}
$$

where $H$ is an entire function on $\mathbf{C}^{2}$ (the Hamiltonian). The local flow $\phi_{t}=\phi(t, \cdot)$ of $X_{H}$ remains in the level sets of $H$ and its Jacobian $J \phi_{t}$ is identically one. Denote by $\Sigma \subset \mathbf{C}^{2}$ the set of critical points of $H$ (i.e., the zeros of $\left.X_{H}\right)$. Then for each $p=\left(z_{0}, w_{0}\right) \in \mathbf{C}^{2} \backslash \Sigma$, the complex orbit $C_{p}$ of $X_{H}$ equals the connected component of the set $\left\{(z, w) \in \mathbf{C}^{2} \backslash \Sigma: H(z, w)=\right.$ $\left.H\left(z_{0}, w_{0}\right)\right\}$ (see [8]). Such components will be called the primary surfaces of $H$.

By connectivity of a Riemann surface $R$ we mean the number of generators of the first homology group $H_{1}(R ; \mathbf{C})$.

1.6 Corollary. If $H$ is an entire function on $\mathbf{C}^{2}$ which has a primary surface of connectivity at least two, then the Hamiltonian vector field $X_{H}$ (3) is not a limit of complete holomorphic vector fields on $\mathbf{C}^{2}$.

Proof. The condition on $H$ implies that $X_{H}$ has a complex orbit of connectivity at least two, and hence this orbit is not one of the surfaces listed in Theorem 1.1.

Example 2. Let $H=w^{2} / 2+Q(z)$, where $Q$ is entire. The corresponding Hamiltonian vector field is

$$
X_{H}(z, w)=w \frac{\partial}{\partial z}+F(z) \frac{\partial}{\partial w}, \quad F=-Q^{\prime} .
$$

This field arises by considering the second order conservative ODE in one complex variable:

$$
\ddot{z}=F(z), \quad z(0)=z_{0}, \quad \dot{z}(0)=\dot{z}_{0},
$$

by introducing the new variable $\dot{z}=w$. If $F$ is affine linear, then $X_{H}$ is affine linear and hence complete. If on the other hand $F$ is not affine linear, then $H$ has primary surfaces of connectivity at least two (see the proof of 
Proposition 7.3 in [8]). Therefore no nonlinear Hamiltonian vector field of type (4) is approximable by complete holomorphic vector fields on $\mathbf{C}^{2}$.

Recall that the complete Hamiltonian holomorphic vector fields on $\mathbf{C}^{2}$ have been classified in [8] (Theorem 6.1); up to conjugation in Aut $\mathbf{C}^{2}$ there are only two types of such fields.

\section{Proof of Theorem 1.1 and Proposition 1.3}

Suppose that $V$ is a locally uniform limit of complete holomorphic vector fields on a Stein manifold $M$. We shall first prove that the maximal domain $\pi_{z}: \Omega_{z} \rightarrow \mathbf{C}$ is single sheeted for each point $z \in M$.

We can embed $M$ as a closed complex submanifold of a Euclidean space $\mathbf{C}^{N}$ [11]. Via this embedding we can think of the flow $\phi(t, z)$ of $V$ as a holomorphic map into $\mathbf{C}^{N}$, and all the usual arguments for holomorphic functions on a Stein manifold apply to $\phi$. Any analytic continuation of the local flow (as a map into $\mathbf{C}^{N}$ ) will remain in the manifold $M$.

Fix $z \in M$ and choose two curves $\gamma_{0}, \gamma_{1} \subset \mathbf{C}$, beginning at 0 and ending at some point $t_{0} \in \mathbf{C}$, such that the local solution $\phi^{z}$ of (1) admits a holomorphic continuation $\phi_{j}^{z}$ along $\gamma_{j}$ for $j=0,1$. If we approximate $V$ in a neighborhood of the compact set $K=\phi_{0}^{z}\left(\gamma_{0}\right) \cup \phi_{1}^{z}\left(\gamma_{1}\right) \subset M$ close enough by a complete holomorphic vector field $W$, then the flow $\psi^{z}$ of $W$ (satisfying $\psi^{z}(0)=z$ ) will exist along $\gamma_{0} \cup \gamma_{1}$, and it will approximate the flow $\phi^{z}$ there. Since $W$ is complete, $\psi^{z}$ extends to a well defined holomorphic map on a strip $R=\{t \in \mathbf{C}:-b<\Im t<a\}$ which contains $\gamma_{0} \cup \gamma_{1}$ ([8], Proposition 2.1). Hence $\psi^{z}\left(t_{0}\right)$ approximates both $\phi_{0}^{z}\left(t_{0}\right)$ and $\phi_{1}^{z}\left(t_{0}\right)$. Since the approximation of $V$ by $W$ can be made arbitrarly close, it follows that $\phi_{0}^{z}\left(t_{0}\right)=\phi_{1}^{z}\left(t_{0}\right)$. This proves that $\Omega_{z}$ is single sheeted, and hence a domain in $\mathbf{C}$.

To prove that $\Omega_{z}$ is simply connected it suffices to show that for every simple closed curve $\gamma \subset \Omega_{z}$ bounding a disc $D \subset \mathbf{C}$ we have $D \subset \Omega$. We connect $\gamma$ to 0 by an arc $\lambda \subset \Omega_{z}$. If $W$ is a complete holomorphic vector field which approximates $V$ in a neighborhood of the compact set

$$
K=\phi^{z}(\lambda \cup \gamma) \subset M
$$

then its flow $\psi^{z}$ (satisfying $\psi^{z}(0)=z$ ) exists and approximates $\phi^{z}$ on $\gamma \cup \lambda$. Since $W$ is complete, the flow $\psi^{z}$ extends holomorphically to a strip $R=\{t \in \mathbf{C}:-b<\Im t<a\} \subset \mathbf{C}$ which contains $\gamma$ and hence $\bar{D}$. Thus $\left.\phi^{z}\right|_{\gamma}$ is a uniform limit of mappings which are holomorphic on $\bar{D}$. Any sequence converging to $\phi^{z}$ on $\gamma$ also converges on $\bar{D}$ by the maximum principle, and it provides a holomorphic extension of $\phi^{z}$ to the disc $D$. Therefore $D \subset \Omega_{z}$.

The statement in Theorem 1.1 concerning the holomorphic type of the complex orbits $C_{z}$ now follows from the following more general result. 
2.1 Proposition. Let $V$ be a holomorphic vector field on a complex manifold $M$, let $\pi_{z}: \Omega_{z} \rightarrow \mathbf{C}$ be the maximal domain of the flow $\phi^{z}$ of $V$ through a point $z \in M$, and let $C_{z}=\phi^{z}\left(\Omega_{z}\right)$ be the complex orbit of $z$. Denote by $\Gamma_{z} \subset$ Aut $\Omega_{z}$ the group of deck transformations of the covering projection $\phi^{z}: \Omega_{z} \rightarrow C_{z}$. Then the projection $\pi_{z}$ induces an isomorphism of $\Gamma_{z}$ onto a discrete subgroup $G_{z} \subset(\mathbf{C},+)$. If $\Omega_{z}$ is simply connected, then the orbit $C_{z}$ is biholomorphic to one of the surfaces $\mathbf{C}, \mathbf{C}^{*}, U$ (=the disc), $U^{*}=U \backslash\{0\}$, an annulus, or a torus.

Remark. The group $G_{z} \subset(\mathbf{C},+)$ described above is called in the literature the 'period group' associated to the orbit $C_{z}$ of the vector field $V$. Denote by $\omega_{z}$ the holomorphic one form on $C_{z}$ which is dual to the vector field $\left.V\right|_{C_{z}}$. In the local coordinate $t$ on $C_{z}$ provided by the map $\phi^{z}$ we have $\omega_{z}=d t$. Then $G_{z} \subset(\mathbf{C},+)$ is the group of all periods of $\omega_{z}$ along closed loops in $C_{z}$.

The first part of the proposition is likely known (and rather trivial); our main purpose is to establish the second part when $\Omega_{z}$ is simply connected.

Proof of Proposition 2.1. We shall first prove the statement about the deck group $\Gamma_{z}$. We consider first the case when the projection $\pi_{z}: \Omega_{z} \rightarrow \mathbf{C}$ is single sheeted, so $\Omega_{z}$ is a domain in C. Fix a $g \in \Gamma_{z}$ and set $a=g(0) \in \Omega_{z}$. We claim that $g$ is the translation $g(t)=t+a$. To see this, observe that both maps $t \rightarrow \phi^{z}(g(t))=\phi^{z}(t)$ and $t \rightarrow \phi^{z}(t+a)$ are local solutions of (1) which pass through the point $z$ at time $t=0$. By uniqueness of the local solution we conclude that $\phi^{z}(g(t))=\phi^{z}(t+a)$ for all $t$ near 0 . Since $\phi^{z}$ is a covering map, it follows that $g(t)=t+a$ as claimed.

In the general case when $\pi_{z}: \Omega_{z} \rightarrow \mathbf{C}$ is not single sheeted, we fix a $g \in \Gamma_{z}$ and consider the holomorphic function $\rho(t)=\pi_{z}(g(t))-\pi_{z}(t)$ for $t \in \Omega_{z}$. The same local argument as above shows that this function is locally constant on $\Omega_{z}$, and hence constant since $\Omega_{z}$ is connected. Thus

$$
\pi_{z}(g(t))=\pi_{z}(t)+\alpha(g), \quad t \in \Omega_{z}
$$

for some constant $\alpha(g) \in \mathbf{C}$. Clearly $g \rightarrow \alpha(g)$ defines a group homomorphism $\alpha: \Gamma_{z} \rightarrow(\mathbf{C},+)$. If $\alpha(g)=0$ for some $g \in \Gamma_{z}$, then $g$ preserves each fiber of $\pi_{z}$. Since the map $\phi^{z}: \Omega_{z} \rightarrow C_{z}$ (which is the maximal solution of (1)) separates points on each fiber of $\pi_{z}$, it follows that $g$ is the identity.

Thus $\alpha$ is an isomorphism of $\Gamma_{z}$ onto the subgroup $G_{z}=\alpha\left(\Gamma_{z}\right) \subset(\mathbf{C},+)$. It follows that the deck group $\Gamma_{z}$ is abelian. Since $\Gamma_{z}$ is discrete, so is $G_{z}$.

Assume from now on that the Riemann surface $\Omega_{z}$ is simply connected and hence biholomorphic either to $\mathbf{C}$ or to the $\operatorname{disc} U$. Then $C_{z}$ is biholomorphic to the quotient $\Omega_{z} / \Gamma_{z}$. 
If $\Omega_{z}$ is biholomorphic to $\mathbf{C}$, then $\Gamma_{z}$ (being a discrete subgroup of $(\mathbf{C},+))$ has zero, one, or two generators. The quotient $C_{z}$ is biholomorphic to, respectively, $\mathbf{C}, \mathbf{C}^{*}$, or a torus.

Suppose now that $\Omega_{z}$ is conformally equivalent to the disc $U$. If $\Gamma_{z}$ is trivial then $C_{z}$ is also the disc. Assume now that $\Gamma_{z}$ is nontrivial. It is known that, if $g_{0}$ and $g_{1}$ are any two automorphisms of the disc which commute, and if $g_{0}$ is not the identity, then $g_{1}$ belongs to the one parameter subgroup generated by $g_{0}[4, \mathrm{p} .76]$. It follows that $\Gamma_{z}$ is a cyclic group with one generator $g$ which is either hyperbolic or parabolic. (The elliptic case is excluded since $\Gamma_{z}$ has no fixed points in $\Omega_{z}$.) In the first case $g$ is conjugate to a dilation $\zeta \rightarrow \lambda \zeta$ on the upper half plane $\Im \zeta>0$ for some $\lambda>0, \lambda \neq 1$, and the quotient $\Omega_{z} / \Gamma_{z}$ is an annulus. In the second case $g$ is conjugate to a translation $\zeta \rightarrow \zeta+\lambda$ on the upper half plane for some $\lambda \in \mathbf{R}, \lambda \neq 0$, and the quotient $\Omega_{z} / \Gamma_{z}$ is the punctured disc.

This concludes the proof of Proposition 2.1.

We continue with the proof of Theorem 1.1. It remains to show that the fundamental domain $\Omega \subset \mathbf{C} \times M$ is pseudoconvex. Choose a smooth strongly plurisubharmonic exhaustion function $\psi: M \rightarrow \mathbf{R}$ and define

$$
\rho: \Omega \rightarrow \mathbf{R} \quad \text { by } \quad \rho(t, z)=|t|^{2}+\psi(z)+\psi(\phi(t, z))
$$

$\rho$ is a smooth strongly plurisubharmonic function on $\Omega$ satisfying

$$
\lim _{t \rightarrow b \Omega_{z}} \rho(t, z)=\infty
$$

for each fixed $z \in M$. The reason is that, as $t$ approaches a finite boundary point of $\Omega_{z}$, the flow $\phi^{z}(t)$ leaves every compact subset of $M$ according to the general properties of flows.

Unfortunately $\rho$ need not be an exhaustion function on $\Omega$, and we need more work to conclude that $\Omega$ is pseudoconvex. It seems worthwhile to state this result independently.

2.2 Proposition. Let $M$ be a Stein manifold and $\pi: M \times \mathbf{C} \rightarrow M$ the projection $\pi(z, t)=z$. Suppose that $\Omega \subset M \times \mathbf{C}$ is a domain satisfying

(i) $\pi(\Omega)=M$,

(ii) for each $z \in M$ the fiber $\Omega_{z}=\{t \in \mathbf{C}:(z, t) \in \Omega\}$ is a connected and simply connected domain containing the origin, and

(iii) there is a plurisubharmonic function $\rho: \Omega \rightarrow \mathbf{R}$ such that for each fixed $z \in M$ and $C \in \mathbf{R}$ the set $\Omega_{z}(C)=\left\{t \in \Omega_{z}: \rho(z, t) \leq C\right\}$ is compact.

Then $\Omega$ is pseudoconvex. 
Remarks. 1. Results of this kind have been proved by Hartogs for several types of geometrically simple fibers $\Omega_{z}$, such as discs and strips in $\mathbf{C}$ (see [3]). In the classical case when $\Omega$ is a Hartogs domain with disc fibers $\Omega_{z}=\{t \in \mathbf{C}:|t|<r(z)\}$, the pseudoconvexity of $\Omega$ is equivalent to the plurisubharmonicity of $-\log r$ on the base $M$.

2. Without the hypothesis (ii) it can still be shown easily that $\Omega$ is pseudoconvex if the base $M$ is one dimensional. We don't know what is the answer in general if we delete (ii).

Proof of Proposition 2.2. Let $U \subset \mathbf{C}$ be the open unit disc. We are going to prove the following stronger property of $\Omega$ : For every analytic disc $F=$ $(f, g): \bar{U} \rightarrow M \times \mathbf{C}$ satisfying $F(b U) \subset \Omega$ we have $F(U) \subset \Omega$. Here $f: \bar{U} \rightarrow$ $M$ and $g: \bar{U} \rightarrow \mathbf{C}$ are holomorphic in a neighborhood of the closed disc $\bar{U}$. Once this is proved, the Kontinuitätssatz implies that $\Omega$ is pseudoconvex. Moreover, in the case $\operatorname{dim}_{\mathbf{C}} \Omega=2$, this also implies the Runge property of $\Omega$ in $M \times \mathbf{C}$.

Fix a disc $F$ as above and define the pull-back domain $D \subset \bar{U} \times \mathbf{C}$ by

$$
D=\left\{(\zeta, w) \in \mathbf{C}^{2}:|\zeta| \leq 1, w \in \Omega_{f(\zeta)}\right\}
$$

Hence $D_{\zeta}=\Omega_{f(\zeta)}$ for $\zeta \in \bar{U}$. The function $\psi: D \rightarrow \mathbf{R}$, defined by $\psi(\zeta, w)=$ $\rho(f(\zeta), w)$, is plurisubharmonic on $D$. For each fixed $\zeta \in \bar{U}$ the function $\psi(\zeta, \cdot)$ exhausts the fiber $D_{\zeta}$, i.e., every sublevel set is compactly contained in $D_{\zeta}$. We have $g(\zeta) \in D_{\zeta}$ for every $|\zeta|=1$, and we must prove that this implies $g(\zeta) \in D_{\zeta}$ for every $\zeta \in U$.

There are several ways to see this. We can use the main result of [6] (Theorem 3) as follows. Choose $\epsilon>0$ sufficiently small such that the disc $\overline{U(0, \epsilon)}=\{w:|w| \leq \epsilon\}$ is contained in every fiber $D_{\zeta}, \zeta \in \bar{U}$. Since the fibers $D_{\zeta}$ are simply connected, we can choose a smooth, one parameter family of manifolds with boundary $Y_{\tau} \subset b U \times \mathbf{C}(0 \leq \tau \leq 1)$, with fibers $Y_{\tau, \zeta}$ which are diffeomorphic to the closed disc, satisfying

(i) $Y_{\tau} \subset Y_{\tau^{\prime}} \subset \subset D$ if $0 \leq \tau \leq \tau^{\prime} \leq 1$,

(ii) $Y_{0, \zeta}=\overline{U(0, \epsilon)}$ and $g(\zeta) \in Y_{1, \zeta}$ for all $|\zeta|=1$,

(iii) the boundaries $T_{\tau}=\left\{(\zeta, w):|\zeta|=1, w \in b Y_{\tau, \zeta}\right\}$ are smooth, totally real, and depend smoothly on $\tau$.

Note that $T_{\tau}$ is a two-dimensional torus which is totally real in $\mathbf{C}^{2}$ if and only if the coordinate projection $T_{\tau} \rightarrow b U$ is a submersion.

The main result of [6] is that, in this setting, the polynomial hulls $K_{\tau}=$ $\hat{Y}_{\tau}=\hat{T}_{\tau}$ also increases continuously (even smoothly) as $\tau$ increases from 0 to 1 . (In fact, the boundary of $K_{\tau}$ is piecewise smooth and consists of two 
Levi flat surfaces, one of them foliated over the base disc $U \times\{0\}$. We shall not need this precise description of the hull.)

Note that $K_{\tau} \subset D$ for small values of $\tau$. Since $\psi$ is uniformly bounded on the family of sets $Y_{\tau}$, the maximum principle implies that $\psi$ remains uniformly bounded on $K_{\tau}=\hat{Y}_{\tau}$ as long as $K_{\tau} \subset D$. Since the fibers $K_{\tau, \zeta}$ increase continuously with $\tau$ for each fixed $\zeta \in U$, and since $\psi(\zeta, \cdot)$ exhausts the fiber $D_{\zeta}$, it follows that $K_{\tau}$ remains in $D$ for all $\tau \in[0,1]$. Since $K_{1}$ contains the graph of $g$, we are done.

An alternative (but essentially equivalent) way to prove that the graph of $g$ is contained in $D$ is as follows. After a small perturbation we may assume that $g(\zeta) \neq 0$ for $|\zeta|=1$. Choose a smooth family of $\operatorname{arcs} \gamma_{\zeta} \subset D_{\zeta}$, $|\zeta|=1$, connecting 0 to $g(\zeta)$ within $D_{\zeta}$, and then 'slide' the disc $g$ along a one parameter family of discs $g_{\tau}: \bar{U} \rightarrow \mathbf{C}(0 \leq \tau \leq 1)$ such that $g_{0}=0$, $g_{1}=g$, and $g_{\tau}(\zeta) \in \gamma_{\zeta}$ for all $|\zeta|=1$ and $0 \leq \tau \leq 1$. Once this is done, the maximum principle applies as above. The required 'sliding' of discs can be done by methods in [6] or in Slodkowski's paper [13].

This concludes the proof of Proposition 2.2 and of Theorem 1.1.

Proof of Proposition 1.3. We may assume that $V(0) \neq 0$ and that its maximal domain $\Omega=\Omega_{0} \subset \mathbf{C}$ is simply connected. Let $\phi: \Omega \rightarrow C=\phi(\Omega) \subset$ $\mathbf{C}$ be the integral curve of $V$ satisfying $\phi(0)=0$. Then $\phi$ is a holomorphic covering map, and since $\Omega$ is simply connected, $\phi$ is the universal covering onto $C$.

We claim that

$$
C=\{z \in \mathbf{C}: V(z) \neq 0\} .
$$

Indeed, $C$ is an open subset of the set where $V \neq 0$, and it suffices to show that $C$ has no boundary in that set.

If $p$ is a boundary point of $C$ where $V(p) \neq 0$, there is a neighborhood $U$ of 0 and a solution $t \rightarrow \phi^{p}(t)$ of the equation (1) for $t \in U$, satisfying $\phi^{p}(0)=p$. The image $\phi^{p}(U)$ is a neighborhood of $p$ and so it contains a point $q \in C \cap \phi^{p}(U)$. Let $q=\phi\left(t_{0}\right)=\phi^{p}\left(t_{1}\right)$, where $\phi$ is as above and $t_{1} \in U$. Set $a=t_{1}-t_{0}$. Then the maps $t \rightarrow \phi(t)$ and $t \rightarrow \phi^{p}(t+a)$ are both solutions of (1), defined for $t$ near $t_{0}$, whose values coincide at $t=t_{0}$. By uniqueness of the local solution they coincide, and we can use the second map to analytically continue $\phi$ to the value $t=t_{0}-t_{1}=-a$ at which we obtain $\phi(t)=\phi^{p}(t+a)=\phi^{p}(0)=q$. This shows that the only boundary points of orbit $C$ are those where $V=0$, and (5) is established.

Since $\Omega$ is assumed to be simply connected, Proposition 2.1 shows that $C$ has connectivity at most one. Hence (5) implies that $V$ has at most one zero in $\mathbf{C}$ and $C$ is equal to either $\mathbf{C}$ or $\mathbf{C}$ minus a point. 
If $V$ has no zeros, then $C=\mathbf{C}$ and hence $\phi: \Omega \rightarrow \mathbf{C}$ is biholomorphic. Thus $\Omega=\mathbf{C}, \phi$ is linear $(\phi(t)=c t)$, and therefore $V(z)=c \frac{\partial}{\partial z}$.

If $V$ has one zero, say $V(c)=0$ for $c \in \mathbf{C}^{*}$, then $C=\mathbf{C} \backslash\{c\}$. In this case we conclude that $\Omega=\mathbf{C}$ and $\phi$ is of the form $\phi(t)=c\left(1-e^{\lambda t}\right)$ for some constant $\lambda \in \mathbf{C}^{*}$. The field $V$ is then affine linear: $V(z)=\lambda(z-c) \frac{\partial}{\partial z}$. This proves Proposition 1.3.

\section{Further examples}

One way to construct holomorphic vector fields on $\mathbf{C}^{n}(n \geq 2)$ which are limits of complete fields, but not necessarily complete, is as follows. Let $F: \mathbf{C}^{n} \rightarrow D \subset \mathbf{C}^{n}$ be a Fatou-Bieberbach map, that is, a biholomorphic map of $\mathbf{C}^{n}$ onto a proper subdomain of $\mathbf{C}^{n}$. Such maps exist in great abundance, see [3] and [12]. In all known constructions of such maps, $F$ arises as a limit of a sequence of holomorphic automorphisms $F_{j} \in \mathrm{Aut}^{n}$. Conversely, if $F: \mathbf{C}^{n} \rightarrow D \subset \mathbf{C}^{n}$ is a Fatou-Bieberbach map onto a Runge domain $D$, then $F$ is a limit of automorphisms of $\mathbf{C}^{n}$ [1], [7]. Moreover, if $J F=1$ (where $J$ denotes the complex Jacobian), then there is an approximating sequence satisfying $J F_{k}=1$.

For every complete holomorphic vector field $W$ on $\mathbf{C}^{n}$, the pull-back field $V(z)=D F^{-1}(w) \cdot W(w)$, where $w=F(z)$, is the limit of the sequence of complete fields $V_{j}=D F_{j}^{-1} \cdot W$. However, $V$ itself is in general not complete since the complex orbits of the field $W$ do not remain inside $D$. In fact we do not know any example of a Fatou-Bieberbach domain which is invariant under the flow of a complete holomorphic vector field.

The earliest and best known construction of such maps is due to Fatou and Bieberbach. We start with an automorphism $\Phi$ of $\mathbf{C}^{n}$ with an attracting fixed point $a$, and we let $D=D(a)$ be the attracting basin

$$
D=\left\{z \in \mathbf{C}^{n}: \lim _{k \rightarrow \infty} \Phi^{k}(z)=a\right\}
$$

Then there exists a biholomorphic map $F: \mathbf{C}^{n} \rightarrow D$ which conjugates $\left.\Phi\right|_{D}$ to an automorphism of $\mathbf{C}^{n}$ which is globally attracting to the origin; see [12]. (In most cases this automorphism is linear.)

Bedford and Smillie [2] proved that, when $D$ is an attracting basin of a polynomial automorphism of $\mathbf{C}^{n}$, then every algebraic curve $\Lambda \subset \mathbf{C}^{n}$ intersects $D$ in a nonempty bounded set. For more general results in this direction we refer the reader to Fornaess and Sibony [5]. If $\Lambda$ is a complex line, then each connected component of $D \cap \Lambda$ is simply connected since $D$ is Runge in $\mathbf{C}^{2}$. In this case the conjugating map $F: \mathbf{C}^{n} \rightarrow D$ has Jacobian one. This gives rise to a number of interesting examples. 
Example 3. There exists a holomorphic vector field $V$ on $\mathbf{C}^{2}$ without critical points such that $V$ is a limit of complete fields, each complex orbit $C_{z}$ $\left(z \in \mathbf{C}^{2}\right)$ is conformally a disc, and the maximal domain $\Omega_{z} \subset \mathbf{C}$ is bounded for each $z \in \mathbf{C}^{2}$.

Indeed, with $F$ as above, it suffices to take $V=D F^{-1} \cdot X$ for any constant vector field $X \neq 0$ on $\mathbf{C}^{2}$ (for example, take $X=\frac{\partial}{\partial z_{1}}$ ). The stated properties of $V$ follows from the result of Bedford and Smillie.

Since $J F=1$, the field $V$ is as well Hamiltonian, and it is a limit of complete Hamiltonian fields.

Example 4. There exists a holomorphic vector field $V$ on $\mathbf{C}^{2}$ which is a limit of complete fields, it has an attracting critical point at the origin 0 , and the basin of attraction of 0 is not all of $\mathbf{C}^{2}$.

To construct such a field, we take $D \subset \mathbf{C}^{2}$ as above to be the attracting basin at 0 of a polynomial automorphism of $\mathbf{C}^{2}$, with the additional property that $\Lambda \cap D$ is disconnected for at least one complex line $\Lambda \subset \mathbf{C}^{2}$ passing through the origin. Such domains can be constructed by using the methods developed in [7] (see in particular Corollary 2.4 in [7]). Indeed, if $D \subset \mathbf{C}^{n}$ is any F-B domain and if $\Lambda$ is any complex line in $\mathbf{C}^{n}$ such that $E=\Lambda \cap D$ is nonempty and bounded, then there is a polynomial automorphism $\Phi \in \operatorname{Aut}^{n}$ such that $\Phi$ is close to the identity near $\bar{E}$, and $\Phi(z) \in \Lambda$ for some point $z \in D \backslash \Lambda$. Then the F-B domain $D^{\prime}=\Phi(D)$ intersects $\Lambda$ in at least two connected components.

Let $X=\lambda \frac{\partial}{\partial z_{1}}+\lambda \frac{\partial}{\partial z_{2}}$ for any constant $\lambda \in \mathbf{C}$ with $\Re \lambda<0$. The flow of $X,(t, z) \rightarrow e^{\lambda t} z$, remains in the complex line through $z$ and 0 , and 0 is a globally attracting point. The field $V=D F^{-1} \cdot X$ on $\mathbf{C}^{2}$ has the required properties. In fact, 0 is an attracting critical point whose basin $B$ consists of all points $z \in \mathbf{C}^{2}$ such that $F(z)$ is connected to 0 in $\Lambda \cap D$, where $\Lambda$ is the complex line through 0 and $F(z)$. Since $\Lambda \cap D$ is not connected for all $\Lambda, B$ is not all of $\mathbf{C}^{2}$.

\section{Acknowledgements}

The proof of the first part of Theorem 1.1 above is based on an idea that was communicated to me (as an example) by G. Buzzard and J. E. Fornæss in January 1995. I wish to thank them for their kind communication. I also thank my colleagues J. P. Rosay and J. Duval for stimulating discussions on this subject.

After the completion of this article I received the paper by Buzzard and Fornæss, entitled 'Complete holomorphic vector fields and time-1 maps', which contains, among other results, a part of our Theorem 1.1. (See Theorem 4.3 in their paper.) 


\section{References}

1. E. Andersén and L. Lempert, On the group of holomorphic automorphisms of $\mathbf{C}^{n}$, Invent. Math. 110 (1992), 371-388.

2. E. Bedford and J. Smillie, Fatou-Bieberbach domains arising from polynomial automorphisms, Indiana Univ. Math. J. 40 (1991), 789-792.

3. S. Bochner and W. T. Martin, Several complex variables, Princeton Univ. Press, Princeton, 1948.

4. L. Carleson and T. Gamelin, Complex dynamics, Springer, New York, 1993.

5. J. E. Fornaess and N. Sibony, Complex dynamics in higher dimension (notes by S. Gavosto), preprint (1994).

6. F. Forstneric, Polynomial hulls of sets fibered over the circle, Indiana Univ. Math. J., 37 (1988), 869-889.

7. F. Forstneric and J. P. Rosay, Approximation of biholomorphic mappings by automorphisms of $\mathbf{C}^{n}$, Invent. Math. 112 (1993), 323-349 ; Correction, Invent. Math. 118 (1994), 573-574.

8. F. Forstneric, Actions of $(\mathbf{R},+)$ and $(\mathbf{C},+)$ on complex manifolds, Math. Z., to appear.

9. J. Globevnik and B. Stensønes, Holomorphic embeddings of planar domains into $\mathbf{C}^{2}$, Math. Ann, to appear.

10. R. C. Gunning and R. Narasimhan, Immersion of open Riemann surfaces, Math. Ann. 174 (1967), 103-108.

11. L. Hörmander, An introduction to complex analysis in several variables, 3rd ed. North-Holland, Amsterdam, 1990.

12. J. P. Rosay and W. Rudin, Holomorphic maps from $\mathbf{C}^{n}$ to $\mathbf{C}^{n}$, Trans. Amer. Math. Soc. 310 (1988), 47-86.

13. Z. Slodkowski, Holomorphic motions and polynomial hulls, Proc. Amer. Math. Soc. 111 (1991), 347-355.

Department of M athematics, University of W isconsin, Madison, W I 53706 E-mail address: forstner@math.wisc.edu 